\title{
Optical polarization shift in beams emitted by quantum cascade lasers
}

\author{
Emilia Pruszyńska-Karbownik ${ }^{1,2}$ (D) Piotr Gutowski ${ }^{2}$ Piotr Karbownik Pa $^{2,3}$
}

Received: 29 April 2019 / Accepted: 19 September 2019 / Published online: 24 September 2019

(c) The Author(s) 2019

\begin{abstract}
In this paper, we present experimental results of current-induced polarization change and transverse mode change in mid-infrared quantum cascade lasers. The polarization of laser beam was determined by measuring of far-field distributions of polarization projection on the linear polarization basis. The measured amount of TE-polarized light is associated with transversal mode structure, which was determined based on the far-field power distributions. The TE polarized light contribution in the beams varies from 6 to $19 \%$. This quantity is anti-correlated to the fundamental transverse mode contribution.
\end{abstract}

Keywords Quantum cascade laser · Polarimetry · Laser beam · Transverse modes · Broadarea lasers

\section{Introduction}

Quantum cascade lasers (QCLs) are unipolar semiconductor devices based on intersubband transition. Because of the polarization selection rule for intersubband transition, the QCLs in principle emit light that is linearly polarized in the epitaxial growth direction (Faist 2013). This property of QCL radiation can be used to reduce the reflectance of the front laser mirror by tilting the mirror (Ahn et al. 2014) instead of using anti-reflective coatings. On the other hand, this is an obstacle in polarization tuning, because the tuning requires the presence of both TM and TE modes (Dhirhe et al. 2013). To achieve high TE-polarizedlight content in QCL beam, Dhirhe et al. designed an asymmetric waveguide as a polarization mode converter which uses birefringence induced by intersubband transitions (Dhirhe

Electronic supplementary material The online version of this article (https://doi.org/10.1007/s1108 2-019-2043-1) contains supplementary material, which is available to authorized users.

Emilia Pruszyńska-Karbownik

ekarb@ite.waw.pl

1 Institute of Physics, Lodz University of Technology, ul. Wólczańska 219, Łódź, Poland

2 Sieć Badawcza Łukasiewicz - Instytut Technologii Elektronowej, al. Lotników 32/46, Warsaw, Poland

3 Development and Implementation Center Telesystem-Mesko Ltd., ul. Warszawska 51, Lubiczów, Poland 
et al. 2012). Also, a photocurrent experiment showed that the selection rule is not absolute and is fulfilled to the accuracy of $3 \%$ (Liu et al. 1998), which also may lead to the occurrence of TE polarized light in QCL beams.

We showed that differently polarized light is present in the beam of quantum cascade lasers with symmetric waveguides (Pruszyńska-Karbownik et al. 2015). A study of another research group showed that in a QCL beam there is a non-negligible amount of circularly polarized light (Janassek et al. 2016). Recently, we have shown that the TE-like polarized light may come from unevennesses of the rear laser mirror (Pruszyńska-Karbownik and Łaszcz 2018). However, the so far presented results concerned only the stable state of polarization and mainly the fundamental transverse mode. Still, studying of polarization for higher-order modes seems perspective, because it was shown that in narrow waveguides some polarization effects are more intensive for higher-order modes than for the fundamental mode (Alexeyev et al. 2018).

In this paper, we present measurement results of beams of multi-transverse-mode midinfrared quantum cascade lasers, in which polarization of radiation changes with supply current. According to our knowledge, this is the first paper concerning the polarization state of broad-area QCL, although this kind of laser is still a subject of extensive research (Heydari et al. 2015; Sergachev et al. 2016; Dente et al. 2017; Mroziewicz and PruszyńskaKarbownik 2019). Likewise, it is the first paper presenting changing of the QCL optical polarization with laser working condition.

\section{Methods}

The studied lasers are quantum cascade lasers with an active region containing $\mathrm{In}_{0.0377} \mathrm{GaAs} / \mathrm{Al}_{0.45} \mathrm{GaAs}$ coupled quantum wells as described in Pierscinska et al. (2018). Laser chips derived from a single epitaxial structure grown by solid source molecular beam epitaxy on a GaAs substrate. Lasers emit light with a wavelength of $9 \mu \mathrm{m}$.

The active region was shaped into $15-\mu \mathrm{m}$ - or $35-\mu \mathrm{m}$-wide double-trench mesa with $\mathrm{Si}_{3} \mathrm{~N}_{4}$ insulation and metal contacts. Figure 1 presents a diagram of so formed waveguide. Detailed information about the technology can be found in Karbownik et al. (2014) and Kosiel et al. (2009).

We have measured far-field profiles of polarization projection on the TE/TM polarization basis. We have used a goniometric profiler (Pruszyńska-Karbownik et al. 2013) with a linear polarizer (Fig. 2). For each current, we have measured two far-field distributions of optical power: with the polarizer in two, perpendicular to each other, positions. The studied

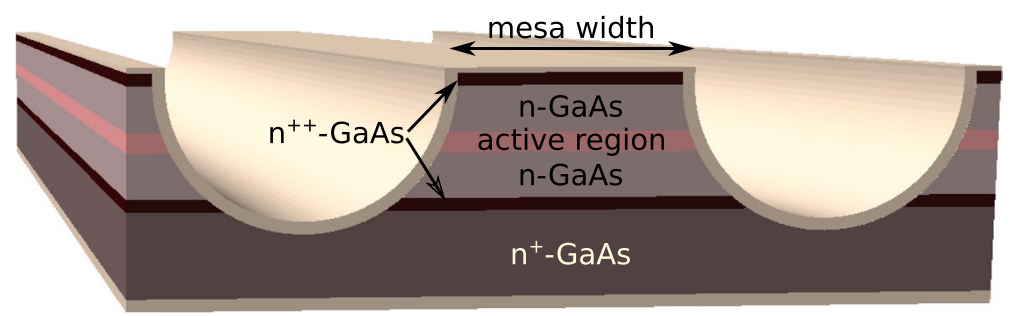

Fig. 1 Waveguide of the studied lasers 
lasers have been supplied with 100-ns pulses and the frequency of $500 \mathrm{~Hz}$. All measurements were performed at room temperature $(293 \mathrm{~K})$.

We performed the measurements without using additional optical elements, like lenses, to avoid polarization by reflection. A check for other unwanted reflections in the experimental set-up was obtained by measuring the far-field in different distances between the laser and the detector. The measured distributions in angular coordinates were equivalent. This means that the source of the entire radiation was in the centre of rotation of the set-up (i.e. the laser front mirror) and the beam did not contain radiation reflected from the set-up components.

Optical power for each polarization and each current was measured by summarizing the far-field power distribution. We calculated the contributions of each polarization as the ratio of the total power value measured for the particular position of the polarizer to the sum of the total power values measured for both positions of the polarizer.

Based on the two-dimensional power distributions, we calculated one-dimensional distributions in the fast axis (i.e. in the epitaxial growth direction) or in the slow axis (i.e. perpendicular to the epitaxial growth direction) by summation over the other axis direction. This simulates one-dimensional slit measurement. Then, we determined the beam diameters $w$ by the second-moment method from the slit data (Wright et al. 1992).

Based on the one-dimensional slow-axis far-field distribution of TM polarized light, we performed reverse beam analysis to achieve intensities of transversal modes in the near field. For this purpose, we used reverse-beam-analysis method based on the effective index method and the least square method. We assume, that in the slow axis the waveguide is homogeneous and confined by metal, what is entitled to double-trench mesas. In this case, the transverse mode distribution on the mirror (i.e. in the near field) takes a sinusoidal form. The transformation from the near field to the far field is calculated by using Huygens principle without the paraxial approximation. The reverse transformation is performed by minimalization the sum of squared differences between the simulated and the measured far-field distributions. We presented a more detailed

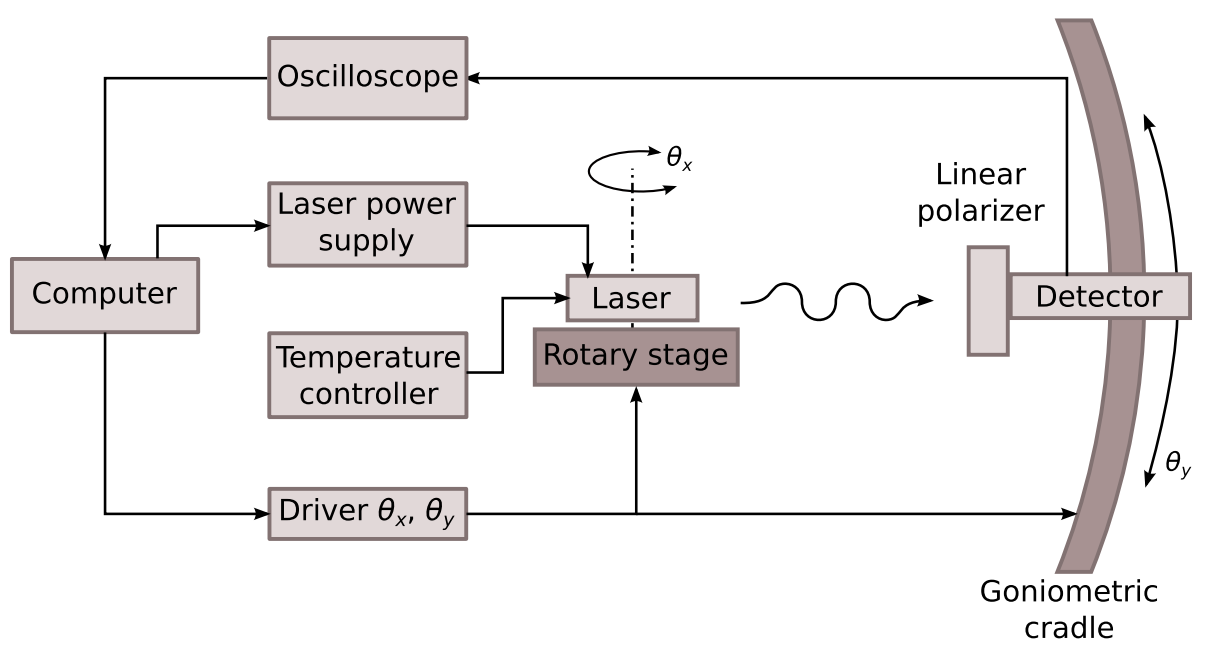

Fig. 2 Diagram of the experimental set-up 
description of the reverse-beam-analysis method with analysis of its correctness and limitations in Pruszyńska-Karbownik et al. (2016).

To analyse correlations between some parameters of the beams, we used $R^{2}$ statistic for linear regression. Coefficient of determination $R^{2}$ for two values $\left(x_{i}, y_{i}\right)$ is defined as

$$
R^{2}=\frac{\Sigma\left(\hat{y}_{i}-\bar{y}\right)^{2}}{\Sigma\left(y_{i}-\bar{y}\right)^{2}},
$$

where $\hat{y}$ is the predicted value of $y$ and $\bar{y}$ is the mean of $y$. In the ideal case, the $R^{2}$ coefficient is equal to 1 . Of course, the $R^{2}$ coefficient for measurement data is always less than one because of measurement uncertainty. The coefficient of determination could be deceptive for non-linear regression, but for linear regression, it is a simple tool to assess the level of correlation (Draper and Smith 1998). In our experiment, we were able to analyse only a small number of measuring points and, therefore, it would be pointless to check other regression than linear. Thus, we accepted the risk, that if the correlation between some parameters are non-linear than we do not notice the correlation. One of the greatest advantages of the $R^{2}$ analysis is its simplicity and, in our opinion, this method is sufficient for the first, initial approach of the problem.

\section{Results and discussion}

Figure 3 presents light-current curves of the studied lasers. Based on the curves, we selected supply currents, for which the optical power is at least $50 \mathrm{~mW}$, so that the impact of noise is minimized. For these currents, we measured the far-field power distributions and polarization rates.

Polarization of the emitted light changed with the current for both lasers. Figure 4 shows graphs of the ratio of the TE-polarized light intensity to the total intensity in the function of the current density. For the laser with wider mesa, the contribution of TE polarized light is considerably higher than for the laser with narrower mesa. In the first case, the maximal contribution of TE polarized light was $19 \%$ for the lowest current and the minimal contribution of TE polarized light was $14.6 \%$ for the current of $13 \mathrm{~A}$. Whereas, in the second case, the contribution of TE polarized light increases with the current: from 6.6 to $7.7 \%$.

Fig. 3 Optical power as a function of current density for the studied quantum cascade lasers. The blue solid line concerns the laser with $15-\mu \mathrm{m}$-wide mesa and the red dotted line concerns the laser with $35-\mu \mathrm{m}$-wide mesa

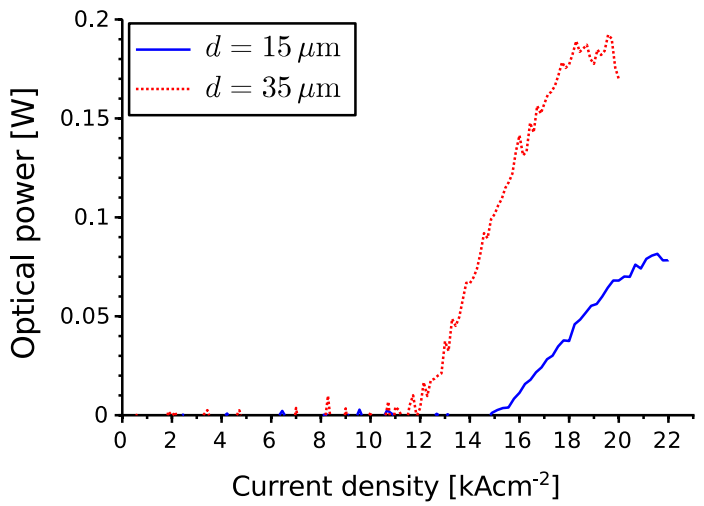


Fig. 4 Measured contribution of TE polarized light in the function of the current density. The experimental data are indicated by symbols. The connecting line is plotted only to improve the clarity of the graph

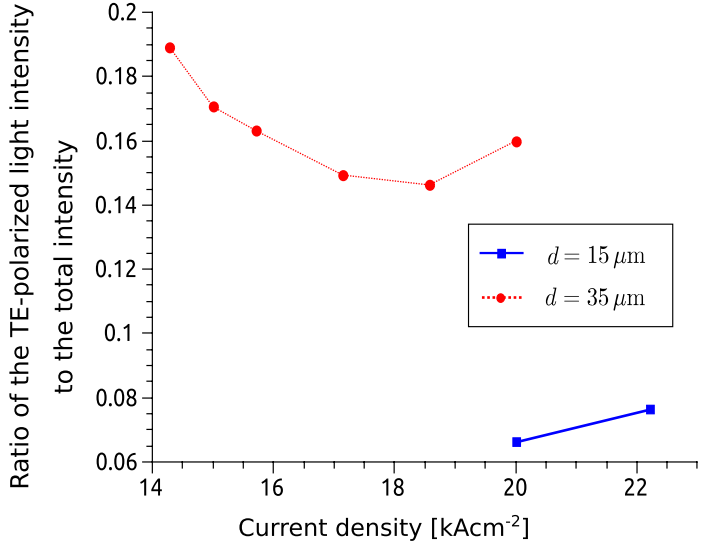

Fig. 5 Far-field power distributions of TM-polarized light in the slow axis for various current density values for the laser with 15- $\mu \mathrm{m}$-wide mesa (a) and for the laser with $35-\mu \mathrm{m}$-wide mesa (b) (a)

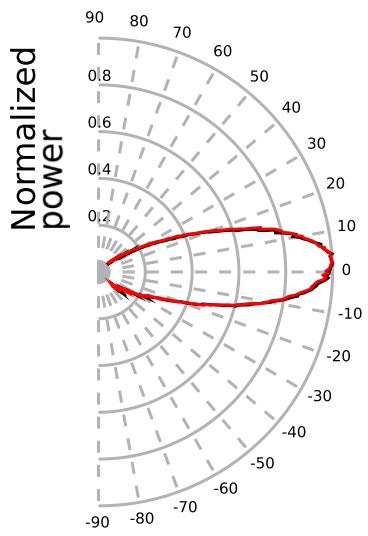

Slow axis angle, degrees

(b)

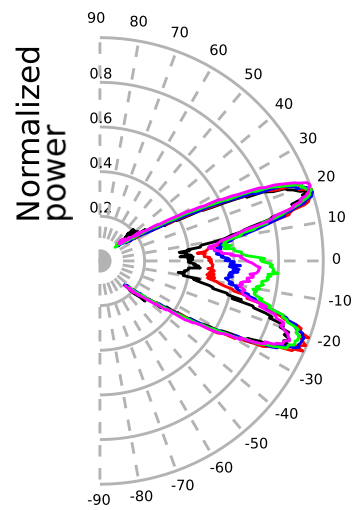
$20.0 \mathrm{kAcm}^{-2}$ $18.6 \mathrm{kAcm}^{-2}$ $17.1 \mathrm{kAcm}^{-2}$ $15.7 \mathrm{kAcm}^{-2}$ $14.3 \mathrm{kAcm}^{-2}$

Slow axis angle, degrees 
The far-field power distributions in the fast axis for both polarizations and the distribution of TE-polarized light in the slow axis were constant to within the noise level. The far-field power distributions of TM-polarized light in the slow axis changed with the current, as shown in Fig. 5. The beam of the wider laser varies with the current and is non-Gaussian - what indicates the presence of higher-order transversal modes-while the beam of the narrower laser seems to be stable and Gaussian. However, in the latter case the determined beam diameter $w$ in the slow axis is narrower than calculated for the fundamental transversal mode in such waveguide: for the lower current $w=32.3^{\circ}$ and for the higher current $w=31.1^{\circ}$, while the theoretical $w=34.0^{\circ}$. Additionally, the beam is tilted by $0.96^{\circ}$ for the lower current and $1.09^{\circ}$ for the higher current. These two facts suggest that, although the fundamental transverse mode is dominant, at least one higher-order transverse mode should be present, as in beam-steering phenomenon (Bewley et al. 2005).

We calculated transverse mode pattern on the laser front mirrors by reverse analysis of the power distributions in the slow axis. We had assumed coexistence of five lowestorder transverse modes: the fundamental mode (0th), the first-order mode (1st), the second-order mode (2nd), the third-order mode (3rd), and the four-order mode (4th). In the case of the $15 \mu \mathrm{m}$-wide mesa assuming only the first three transverse modes is sufficient to obtain a good fitting to the measurement results. Figure 6 presents the calculated intensities of the modes in the function of the supply current density.

In the case of the laser with narrower mesa, for both supply current values, the dominant mode is the fundamental one. The contribution of the fundamental mode decreases with the current, which is accompanied by increasing of TE-polarized light contribution.

In the case of the laser with the wider mesa, in the whole range of current, the dominant transverse modes are: the fundamental mode, the second-order mode, and the third-order mode and these modes compete with each other. The shares of the remaining modes (the first-order and the fourth-order modes) are below $10 \%$.

Because both the TE-polarized light contribution in the beam and the transverse mode structure change with current, we checked a correlation between the TE-polarized light contribution and the contributions of individual transversal modes. Table 1 presents the calculated values of the statistical coefficient of determination $R^{2}$ and the type (positive or negative) of all these correlations. We calculated values of $R^{2}$ for two sets of parameters:

Fig. 6 Relative intensities of lowest-order transversal modes calculated by reverse analysis of the laser beam, in the function of the supply current density. The experimental data are indicated by symbols: filled symbols concern the narrower laser $(d=15 \mu \mathrm{m})$ and empty symbols concern the wider laser $(d=35 \mu \mathrm{m})$. The lines are plotted only to improve the clarity of the graphs

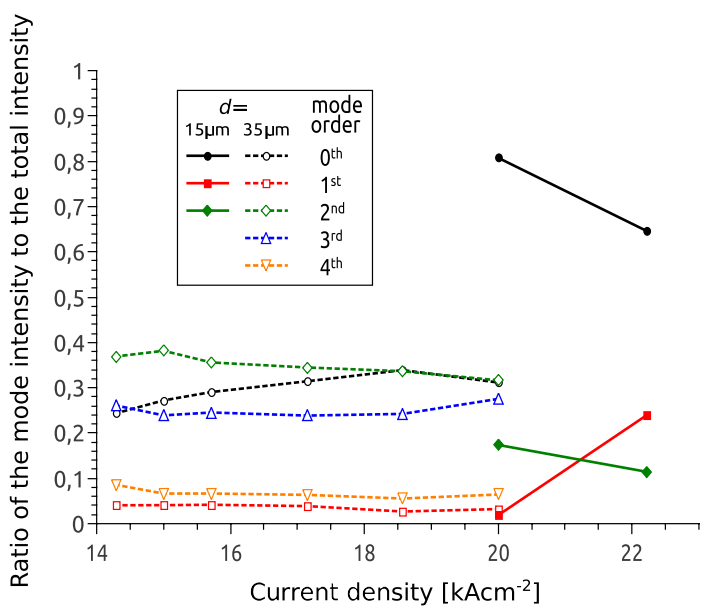


Table 1 Values of the coefficient of determination $R^{2}$ and the correlation types of linear regression between the contribution of TE-polarized light in the beam and the contribution of the transverse modes in the laser resonator

\begin{tabular}{llllll}
\hline $\begin{array}{l}\text { Transverse } \\
\text { mode order }\end{array}$ & \multicolumn{2}{l}{ For $35 \mu \mathrm{m}$-wide laser } & & \multicolumn{2}{l}{ For both lasers } \\
\cline { 2 - 3 } \cline { 5 - 5 } 0th & $R^{2}$ & Type of correlation & & $R^{2}$ & Type of correlation \\
1st & 0.941 & Negative & & 0.959 & Negative \\
2nd & 0.373 & Positive & & 0.434 & Negative \\
3rd & 0.392 & Positive & 0.898 & Positive \\
4th & 0.125 & Positive & & \\
\hline
\end{tabular}

collected only for the wider laser $(d=35 \mu \mathrm{m})$ and collected for both lasers together. We did not calculate that for the narrower laser alone, because only two measurement points fulfilled the condition of sufficient power and in this case the determination of $R^{2}$ is pointless.

\section{Conclusions}

In this paper, we have shown that not only the optical polarization of a quantum cascade laser is not necessarily linear but it can also change with the laser's working conditions.

There is a very strong $\left(R^{2}>0.9\right)$ negative correlation between TE-polarized light contribution and the fundamental mode contribution, which means that if the fundamental mode contribution decreases the TE-polarized light contribution increases. This could indicate a relationship between the polarization of the beams of the quantum cascade lasers and the presence of higher-order modes in their transverse mode structure.

Acknowledgements This work was supported by Project 2015/19/N/ST7/01203 funded by the National Science Centre, Poland.

Open Access This article is distributed under the terms of the Creative Commons Attribution 4.0 International License (http://creativecommons.org/licenses/by/4.0/), which permits unrestricted use, distribution, and reproduction in any medium, provided you give appropriate credit to the original author(s) and the source, provide a link to the Creative Commons license, and indicate if changes were made.

\section{References}

Ahn, S., Ristanic, D., Gansch, R., Reininger, P., Schwarzer, C., MacFarland, D.C., Detz, H., Schrenk, W., Strasser, G.: Quantum cascade lasers with a tilted facet utilizing the inherent polarization purity. Opt. Express 22(21), 26294-26301 (2014). https://doi.org/10.1364/OE.22.026294

Alexeyev, C.N., Alexeyeva, M.C., Lapin, B., Vikulin, D., Yavorsky, M.: Polarization plane rotation for higher order modes in twisted optical fibers with discrete rotationally symmetric core. IOP Conf. Ser. J. Phys. Conf. Ser. 1124, 051006 (2018). https://doi.org/10.1088/1742-6596/1124/5/051006

Bewley, W.W., Lindle, J.R., Kim, C.S., Vurgaftman, I., Meyer, J.R., Evans, A.J., Yu, J.S., Slivken, S., Razeghi, M.: Beam steering in high-power CW quantum-cascade lasers. IEEE J. Quantum Electron. 41(6), 833-841 (2005)

Dente, G.C., Tilton, M.L.: Lateral mode constrictions for broad-ridge quantum cascade lasers. Appl. Opt. 56(18), 5164-5166 (2017). https://doi.org/10.1364/AO.56.005164

Dhirhe, D., Slight, T., Holmes, B., Hutchings, D., Ironside, C.: Quantum cascade lasers with an integrated polarization mode converter. Opt. Express 20(23), 25711-25717 (2012). https://doi.org/10.1364/ OE.20.025711 
Dhirhe, D., Slight, T.J., Holmes, B.M., Ironside, C.N., International, H., Park, T.: Active polarisation control of a quantum cascade laser using tuneable birefringence in waveguides. Opt. Express 21(20), 2426724280 (2013). https://doi.org/10.1364/OE.21.024267

Draper, N., Smith, H.: Applied Regression Analysis. Wiley Series in Probability and Statistics. Wiley, New York (1998)

Faist, J.: Quantum Cascade Lasers. OUP Oxford, Oxford (2013)

Heydari, D., Bai, Y., Bandyopadhyay, N., Slivken, S., Razeghi, M.: High brightness angled cavity quantum cascade lasers. Appl. Phys. Lett. 106(9), 091105 (2015). https://doi.org/10.1063/1.4914477

Janassek, P., Hartmann, S., Molitor, A., Michel, F., Elsäßer, W.: Investigations of the polarization behavior of quantum cascade lasers by Stokes parameters. Opt. Lett. 41(2), 305-308 (2016)

Karbownik, P., Trajnerowicz, A., Szerling, A., Wójcik-Jedlińska, A., Wasiak, M., Pruszyńska-Karbownik, E., Kosiel, K., Gronowska, I., Sarzała, R., Bugajski, M.: Direct Au-Au bonding technology for high performance GaAs/AlGaAs quantum cascade lasers. Opt. Quant. Electron. 47, 893-899 (2014). https ://doi.org/10.1007/s11082-014-0031-z

Kosiel, K., Szerling, A., Kubacka-Traczyk, J., Karbownik, P., Pruszyńska-Karbownik, E., Bugajski, M.: Molecular beam epitaxy growth for quantum cascade lasers. Acta Phys. Pol. A 116(5), 806-813 (2009)

Liu, H., Buchanan, M., Wasilewski, Z.: How good is the polarization selection rule for intersubband transitions? Appl. Phys. Lett. 72(14), 1682-1684 (1998)

Mroziewicz, B., Pruszyńska-Karbownik, E.: On the beam radiance of mid-infrared quantum cascade lasers: a review. Opto-Electron. Rev. 27, 161-173 (2019). https://doi.org/10.1016/j.opelre.2019.05.001

Pierscinska, D., Gutowski, P., Haldas, G., Kolek, A., Sankowska, I., Grzonka, J., Mizera, J., Pierscinski, K., Bugajski, M.: Above room temperature operation of InGaAs/AlGaAs/GaAs quantum cascade lasers. Semicond. Sci. Technol. 33, 035006 (2018)

Pruszyńska-Karbownik, E., Regiński, K., Mroziewicz, B., Szymański, M., Karbownik, P., Kosiel, K., Szerling, A.: In: Tenth Symposium on Laser Technology (International Society for Optics and Photonics, 2013), pp. 87020E-87020E

Pruszyńska-Karbownik, E., Trajnerowicz, A., Karbownik, P., Gutowski, P., Regiński, K., Bugajski, M.: In: 3rd Annual Conference on COSTActionMP1204 and 6th International Conference on Semiconductor Mid-IR Materials and Optics SMMO (2015)

Pruszyńska-Karbownik, E., Regiński, K., Bugajski, M.: A novel method to calculate a near field of widely divergent laser beams. Opt. Quant. Electron. 48(5), 301 (2016)

Pruszyńska-Karbownik, E., Łaszcz, A.: In: 2018 International Conference Laser Optics (ICLO) (IEEE, 2018)

Sergachev, I., Maulini, R., Bismuto, A., Blaser, S., Gresch, T., Muller, A.: Gain-guided broad area quantum cascade lasers emitting 23.5 W peak power at room temperature. Opt. Express 24(17), 19063-19071 (2016). https://doi.org/10.1364/OE.24.019063

Wright, D., Greve, P., Fleischer, J., Austin, L.: Laser beam width, divergence and beam propagation factoran international standardization approach. Opt. Quant. Electron. 24(9), S993-S1000 (1992)

Publisher's Note Springer Nature remains neutral with regard to jurisdictional claims in published maps and institutional affiliations. 